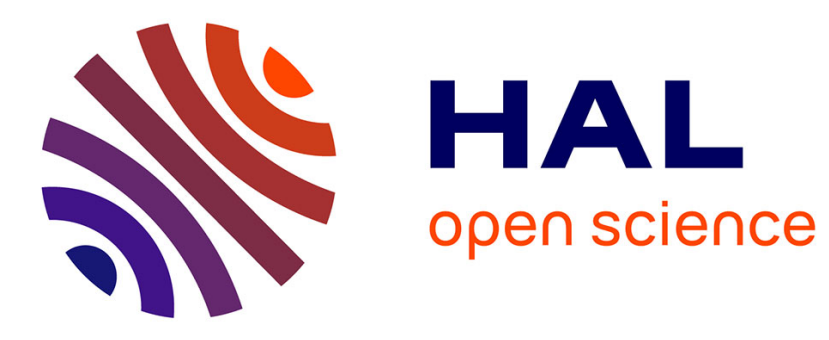

\title{
Method of analysing non-stationary electrical signals
}

Georgia Cablea, Pierre Granjon, Christophe Bérenguer

\section{To cite this version:}

Georgia Cablea, Pierre Granjon, Christophe Bérenguer. Method of analysing non-stationary electrical signals. CMMNO 2014 - 4th International Conference on Condition Monitoring of Machinery in NonStationary Operations, Dec 2014, Lyon, France. hal-01103191

\section{HAL Id: hal-01103191 \\ https://hal.science/hal-01103191}

Submitted on 14 Jan 2015

HAL is a multi-disciplinary open access archive for the deposit and dissemination of scientific research documents, whether they are published or not. The documents may come from teaching and research institutions in France or abroad, or from public or private research centers.
L'archive ouverte pluridisciplinaire HAL, est destinée au dépôt et à la diffusion de documents scientifiques de niveau recherche, publiés ou non, émanant des établissements d'enseignement et de recherche français ou étrangers, des laboratoires publics ou privés. 


\title{
Method of analysing non-stationary electrical signals
}

\author{
Georgia Cablea, Pierre Granjon and Christophe Bérenguer \\ Gipsa-lab, Grenoble University, France \\ \{georgia.cablea, pierre.granjon, \\ christophe.berenguer\}@gipsa-lab.grenoble-inp.fr
}

\begin{abstract}
Considering the non-stationary operating conditions of wind turbines, electrical signals measured at the stator of their generators will also present variations around their fundamental frequency. This paper presents a method able to efficiently analyse electric quantities measured at the generator stator. The obtained outputs consist in electrical features that fully describe the electrical information contained in the measured three-phase quantities. These features can be directly used or further analysed to obtain efficient fault indicators. The proposed method relies on using the instantaneous symmetrical components to describe the quantities and complex-valued filtering to select the content around the fundamental frequency. The obtained sample per sample algorithm can be implemented on-line, and is able to process stationary or nonstationary quantities in order to extract the useful information around the fundamental frequency. The performance of the proposed method, as well as its capability to detect mechanical faults, is illustrated using experimental data.
\end{abstract}

Keywords: condition monitoring $\cdot$ wind turbine $\cdot$ tree-phase electrical signals $\cdot$ non-stationary signals $\cdot$ electrical signature analysis

\section{Introduction}

In terms of renewable energy, wind farms provide a promising mean to extract energy from the wind. Wind turbines, whether they are onshore or offshore, are still a developing technology [1]. Though their reliability has improved in time, it can be further increased by implementing efficient condition monitoring systems and predictive maintenance strategies. Currently, vibration analysis seems to be the most popular condition monitoring technique [2] for such systems. Nonetheless, while vibration analysis focuses on mechanical faults, it has been shown [1] that the electrical sub-assemblies (generator, converter, etc.) are also critical components in wind turbines. In terms of monitoring the electrical subassemblies, using the signals measured at the output of the generator represents the obvious solution. The main challenge in monitoring wind turbines is that they often run under non-stationary operating conditions because of wind speed fluctuations. This drastically limits the amount of stationary data generally used 
by classical condition monitoring systems. An efficient CMS must then be able to manage and analyse such non-stationary data in order to generate efficient fault indicators. Electrical signals measured at the stator of a wind turbine generator contain most information around the electrical fundamental frequency. Considering the non-stationary operating conditions of the turbine, electrical signals will also present variations around their fundamental frequency. Nonetheless, these variations are smaller than, for example, variations in the vibrations signals.

In this paper, the proposed method relies on using the instantaneous symmetrical components [3] to describe the quantities and complex-valued filtering [4] to select the content around the fundamental frequency. The method of symmetrical components decompositions is a widely used method in the fields of power network calculations [3] (network modelling and control, power quality monitoring, fault detection, etc) and monitoring of three-phase electrical machines [5]. More recently, an increased interest is shown towards monitoring mechanical faults in the drive trains using electrical quantities measured at the stator of three-phase electrical motors [6] [7] and generators [8] [9].

Section 2 presents the considered signals model and the method for estimating the electrical quantities. A brief overview of the whole algorithm is then provided in Section 2.2. The performance of the proposed method and its ability to be used in mechanical fault detection applications is presented in Section 3 using experimental signals obtained from a test-bench for a wind turbine.

\section{Method for Electrical Signature Analysis}

\subsection{Model and Features of Three-Phase Signals}

The three-phase electrical signals (currents and voltages) are generically denoted by $x$ in what follows. Such signals mainly consist in a fundamental component of frequency $f_{0}$, with possible amplitude and frequency modulations, some harmonics and additive noise. Therefore they are usually analysed around their fundamental frequency. Equation (1) gives a simple analytical signal model of three-phase signals around $f_{0}$. The relation between the real signal and the analytical one is given by $x(t)=\Re\{\underline{x}(t)\}$.

$$
\left[\begin{array}{l}
\underline{x}_{1}(t) \\
\underline{x}_{2}(t) \\
\underline{x}_{3}(t)
\end{array}\right]=\left[\begin{array}{c}
X_{1}(t) \\
X_{2}(t) e^{-j\left(\frac{2 \pi}{3}+\rho_{1}(t)\right)} \\
X_{3}(t) e^{-j\left(\frac{4 \pi}{3}+\rho_{2}(t)\right)}
\end{array}\right] e^{j 2 \pi \int_{0}^{t} f_{0}(u) \mathrm{d} u}+\left[\begin{array}{c}
n_{1}(t) \\
n_{2}(t) \\
n_{3}(t)
\end{array}\right]
$$

In equation (1) the signal on phase 1 is considered the reference signal and the quantities described around $f_{0}$ are:

- $\underline{x}_{1}(t), \underline{x}_{2}(t)$ and $\underline{x}_{3}(t)$ representing the three-phase signals;

- $X_{1}(t), X_{2}(t)$ and $X_{3}(t)$ representing the instantaneous magnitudes of the signals and containing possible amplitude modulations;

- $\rho_{1}(t)$ and $\rho_{2}(t)$ representing the small errors in phase shifts between phases;

- $f_{0}(t)$ representing the instantaneous frequency of the components and containing eventual frequency modulations; 
- $n_{1}(t), n_{2}(t)$ and $n_{3}(t)$ representing additive noise.

The magnitude and frequency modulations may be due to changes in the operating point of the system or to fault occurrence (i.e. mechanical faults in rotating machines [7], broken rotor bar [10], bearing faults [5], etc.).

Perfectly balanced three-phase quantities would have the same amplitude on all phases and a phase shift of $\frac{2 \pi}{3}$ between each of the phases. This corresponds to $X_{1}(t)=X_{2}(t)=X_{3}(t)$ and $\rho_{i}(t)=0$. However, real systems are never perfectly balanced. The electrical unbalance is generally due to faults in the three-phase systems (i.e. winding stator faults in rotating machines [11]). One way to separate any three-phase unbalanced system into balanced components is to apply the Lyon transform [12], [3], [13].

$$
\left[\begin{array}{l}
\underline{x}_{+}(t) \\
\underline{x}_{-}(t) \\
\underline{x}_{0}(t)
\end{array}\right]=\frac{2}{3}\left[\begin{array}{ccc}
1 & a & a^{2} \\
1 & a^{2} & a \\
1 & 1 & 1
\end{array}\right]\left[\begin{array}{l}
\underline{x}_{1}(t) \\
\underline{x}_{2}(t) \\
\underline{x}_{3}(t)
\end{array}\right]
$$

Where:

- $a=e^{j \frac{2 \pi}{3}}$, called the Fortescue [14] operator, represents a phase shift of $\frac{2 \pi}{3}$;

- $\underline{x}_{+}(t), \underline{x}_{-}(t)$ and $\underline{x}_{0}(t)$ are generically called instantaneous symmetrical components and are the positive-, negative- and zero-sequence components respectively.

Considering equation (1), which describes the content of the measured signal around $f_{0}$, and applying the transformation from equation (2) to split the signal into its balanced and unbalanced parts, the instantaneous symmetrical components of the system around $+f_{0}$ are obtained. At each frequency, the instantaneous positive-sequence component describes the balanced quantities in the system, whereas the instantaneous negative- and zero-sequence ones quantify the amount of unbalance in the system for the respective frequencies [6]. While depending on the system configuration $x_{0}(t)$ may not always be related to a fault (i.e. in four wire connections $i_{0}(t)$ is related to the neutral current), in what follows the more generic scenario in which the zero-sequence component quantifies faults to ground is considered.

The linear transformation of equation (2) applied to the mono-component signals of equation (1) leads to the mono-component signals of $\underline{x}_{+}(t), \underline{x}_{-}(t)$ and $\underline{x}_{0}(t)$, neglecting the noise. Using the instantaneous symmetrical components one can demodulate the amplitude and frequency [15] [13] as:

$$
\begin{gathered}
X_{+}(t)=\left|\underline{x}_{+}(t)\right| \quad X_{-}(t)=\left|\underline{x}_{-}(t)\right| \quad X_{0}(t)=\left|\underline{x}_{0}(t)\right| \\
f_{x_{+}}(t)=\frac{1}{2 \pi} \frac{d \theta_{+}(t)}{d t} \quad, \text { with: } \theta_{+}(t)=\underline{x}_{+}(t)
\end{gathered}
$$

In order to quantify the amount of electrical unbalance in a system, one can use the advantage provided by the use of symmetrical components, that is 
the separation into balanced and unbalanced quantities. Using the magnitudes of such components, an electrical unbalance indicator can be defined as in (5). Regarding the indicator presented in equation (5), it should be further emphasised that it only characterises the electrical unbalance around the fundamental frequency $f_{0}[13]$.

$$
u_{\underline{x}}(t)=\sqrt{\frac{\left|\underline{x}_{-}(t)\right|^{2}+\left|\underline{x}_{0}(t)\right|^{2}}{\left|\underline{x}_{+}(t)\right|^{2}+\left|\underline{x}_{-}(t)\right|^{2}+\left|\underline{x}_{0}(t)\right|^{2}}}
$$

If both currents and voltages are available, instantaneous three-phase electrical powers can also be estimated using the instantaneous positive-sequence components of the voltages and currents as:

$$
\begin{array}{cl}
\text { Complex power : } & \underline{p}_{+}(t)=\frac{3}{2} \underline{v}_{+}(t) \underline{i}_{+}^{*}(t) \\
\text { Active power : } & \underline{P}_{+}(t)=\Re\left\{\underline{p}_{+}(t)\right\} \\
\text { Reactive power : } & \underline{Q}_{+}(t)=\Im\left\{\underline{p}_{+}(t)\right\} \\
\text { Apparent power : } & \underline{S}_{+}(t)=\left|\underline{p}_{+}(t)\right| \\
\text { Power factor : } & \cos \left(\varphi_{0}\right), \text { where } \varphi_{0}=/ \underline{p}_{+}(t)
\end{array}
$$

\subsection{Estimation Algorithm}

In order to summarize the steps needed to implement this method, the estimation algorithm has been graphically represented in Figure 1a for the computed quantities for the voltages and currents independently and in Figure 1b for computing the electrical power related quantities using the positive sequence components. In this section the algorithm is briefly described, while more details are provided in $[13]$.

In Figure 1a, at the input of the algorithm there are the electrical three-phase quantities. The first step of the algorithm is a down-sampling step. Considering that the fundamental frequency of electrical signals is not a high frequency and in order to save computation time this step may be implemented if needed, depending on the sampling frequency used by the acquisition system. In order to select the frequency content around the fundamental, a complex-valued bandpass filter [4] is used. The filter is implemented as a finite impulse response filter with complex-valued coefficients. Because of the linear time-invariant characteristic of the filter and of the symmetrical components transformation, these operations can and have been switched in the implementation of the algorithm. In the end, the instantaneous symmetrical components are used to compute the electrical unbalance indicator. The positive sequence component is also used for the amplitude and frequency demodulation step, thus obtaining the instantaneous magnitude and frequency of the positive sequence component around the fundamental frequency. 
Figure 1b graphically depicts the algorithm for the estimation of the electrical powers related features computed using the positive sequence components of the voltages and currents. As emphasised by the figure, the operations used for these estimations are simple and thus low time-consuming making this algorithm appropriate for embedded implementations.

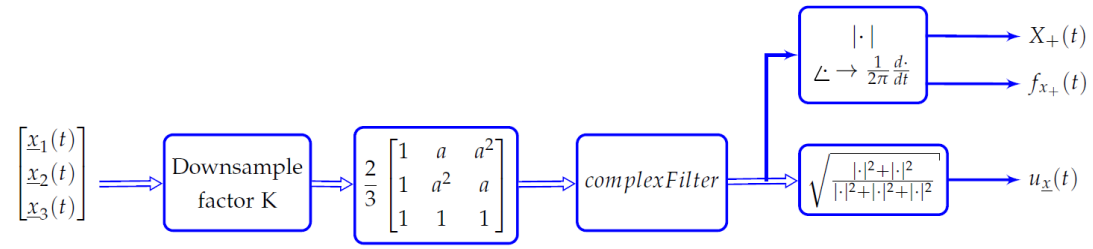

(a) Algorithm estimating the electrical three-phase quantities

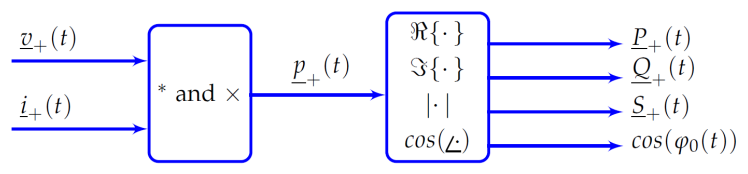

(b) Algorithm estimating the electrical powers features

Fig. 1: Estimation algorithm

Considering that a filter is used to select the bandwidth around the fundamental frequency of the electrical signals, if the frequency varies too much it may get out of the assigned bandwidth. In most real wind turbine systems however, the generator output would be connected to the main grid, which will impose a constant fundamental frequency. The output signals frequency would not be allowed to vary too much, if at all. Another possible way to overcome this limitation could also be to use an adaptive filtering approach for the complex bandpass filter.

\section{$3 \quad$ Experimental Results}

An experimental test-bench has been developed in the CETIM laboratory in Senlis, France. This bench emulates the structure and behaviour of a wind turbine. The structure is presented in Figure 2a. The operating conditions are determined by controlling the speed of the low speed shaft, which is considered to be the system input. For this paper, the electrical signals are acquired at the output of the generator. An experiment was conducted for $\approx 200$ hours during which an accelerated deterioration of the main bearing was induced by applying axial and radial forces on the main bearing. Throughout the experiment several operating conditions were alternated. In this paper, two of the operating states are considered. Presented in Figure 2b, State A denotes the stationary conditions while State B uses non-stationary input signals. All other operating parameters for the system are the same for both states. At the end of the experiment, an inner race fault was observed in the main bearing. 


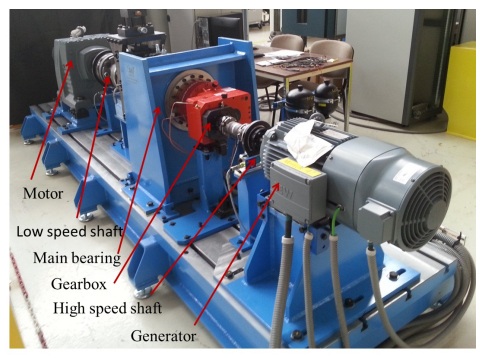

(a) Experimental test bench

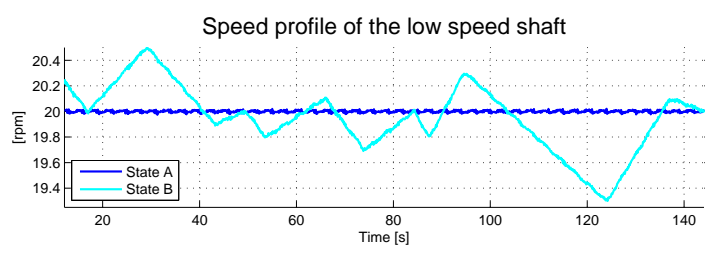

(b) Speed profile of the low speed shaft

Fig. 2: Experimental set-up

\subsection{Electrical Indicators Behaviour}

In what follows, the signals used have been recorded at $\approx 19$ hours into the experiment for a duration of $\approx 145$ for state $\mathrm{A}$ and state $\mathrm{B}$, consequently. The goal of this section is to present the capability of the computed indicators to estimate and track the evolution of the electrical quantities and to compare their behaviour in stationary and non-stationary conditions. For this purpose, the chosen measurements are the voltages. Nonetheless, similar results have been obtained also by using the currents and the estimated electrical powers.

For the non-stationary signal the time-frequency representation (spectrogram) of the voltage positive sequence component is presented in Figure 3. The evolution of the fundamental frequency of the voltages can be observed in the figure. The profile of the fundamental frequency of this estimated quantity bears a close resemblance to the speed profile of the low speed shaft presented in Figure $2 \mathrm{~b}$. The variations of the instantaneous amplitude and frequency of the fundamental component contain information related to changes in the operating conditions as well as to mechanical phenomena occurring in the system, hence the need for demodulations.

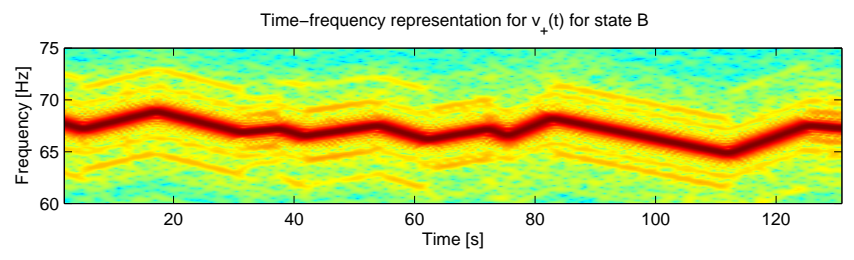

Fig. 3: Time-frequency representation around the fundamental frequency of the voltage positive sequence component for non-stationary conditions

Figure 4a depicts the estimation of the instantaneous fundamental frequency of the electrical signals, computed using the voltage positive sequence component. While for state A the value of the estimation is almost constant, for state $\mathrm{B}$ the time evolution of the frequency bears a close resemblance to the speed variations of the low speed shaft (see Figure 2b), as expected. Figure $4 \mathrm{~b}$ depicts 
the instantaneous magnitude estimated for the voltage positive sequence components computed around the fundamental frequency $f_{0}$. While for the stationary case in state A this magnitude remains almost constant, for the non-stationary case of state B the magnitude is obviously influenced by the variation of the rotating speed imposed at the input of the system.

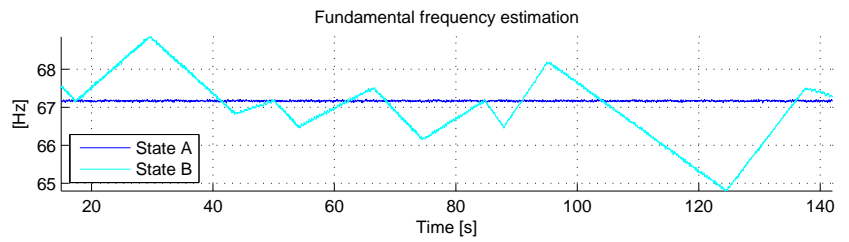

(a) Estimation of the fundamental frequency of $v_{+}(t)$

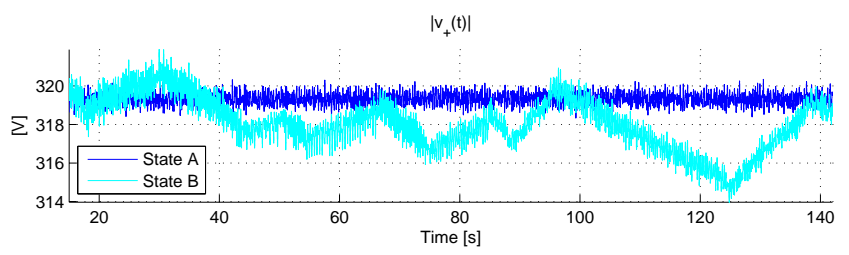

(b) Amplitude of the voltages positive sequence component

Fig. 4: Frequency and amplitude modulations of the positive sequence component of the voltages

Figure 5 depicts the results obtained for the unbalance indicator computed for the voltages. While there was no electrical unbalance present in the system throughout the whole experiment, the goal of presenting this result is to show that this indicator is not influenced by the non-stationary conditions in state B. While theoretically the value of this indicator should be 0 in case of electrically balanced systems, in practice real systems are not perfectly balanced. This explains the small value greater than null that this indicator presents.

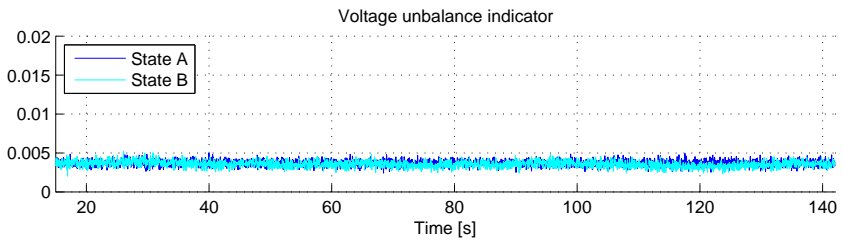

Fig. 5: Voltage unbalance indicator computed around $f_{0}$

In this section, the performance of the proposed indicators was illustrated using the experimental results obtained using the voltages measurements. The presented results show good performance in terms of estimating and tracking electrical feature. Moreover, it has been shown that the unbalance indicator is not affected by the non-stationary operating conditions. Similar results have been obtained also for the currents and electrical power estimations. 


\subsection{Mechanical Fault Detection Capabilities}

The goal of the performed experiment was to induce accelerated deterioration of the main bearing and to study the fault signature on various measured signals. At the end of the test, an inner race bearing fault occurred in the main bearing. This fault was confirmed by physical inspection after the experiment was finished. The fault frequency is known to be $f_{f}=3.4506 \mathrm{~Hz}$ and the start of its evolution was determined by using vibration signals acquired by accelerometers placed on the respective bearing. For this section, three sets of signals will be considered before the fault and three sets after the fault occurred, for both stationary and non-stationary conditions.

Figures 6a and 6b present the power spectral densities (PSDs) (using Welch's method [16]) computed for the magnitude of the positive sequence component of the voltages. Figure 6a depicts these PSDs computed under the stationary conditions in state A. The blue curves show the results of the sets before the fault occurred while the red profiles show the resulting PSDs after the inner race bearing fault occurred. As it can be seen in the figure, after the fault a new peak appears at the fault frequency $f_{f}$. This fault frequency is also modulated by the rotating frequency of the low speed shaft, thus two smaller peaks can be observed at $f_{f}+f_{r o t}$ and $f_{f}-f_{\text {rot }}$. The other peaks present in the PSD of the magnitude of the voltage positive sequence component in Figure 6a are accounted for by mechanical phenomena. For example, the peak located at $2.33 \mathrm{~Hz}$ corresponds to the epicyclic gear train frequency computed for a rotating speed of the low speed shaft of $20 \mathrm{rpm}$.

Figure $6 \mathrm{~b}$ presents the same results computed for state B. By comparing to Figure $6 \mathrm{a}$, one can observe that mainly the same peaks are present. However, in the non-stationary case the peaks in the PSD are not as high and they are wider. It is worth emphasising that this approach of analysing the spectral content of the signals may not be the optimum in terms of estimation performance, but it can be observed that for small variations in the signals, it can be used as a rough estimator. Due to the fact that the rotating frequency is varying, the modulations induced by the fault are not as clear as in the stationary case, but they are visible nonetheless.

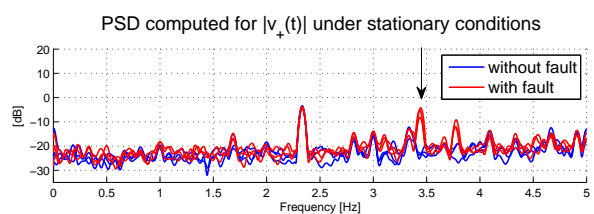

(a) PSD of $\left|v_{+}(t)\right|$ for state $\mathrm{A}$

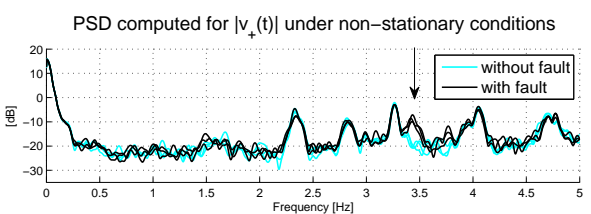

(b) PSD of $\left|v_{+}(t)\right|$ for state $\mathrm{B}$

Fig. 6: PSD of $\left|v_{+}(t)\right|$ for stationary and non-stationary operating conditions

Figures $7 \mathrm{a}$ and $7 \mathrm{~b}$ depict the PSDs computed for the estimated fundamental frequency of the positive sequence component of the voltages. As for the magni- 
tude indicator, the frequency indicator also shows a peak at the fault frequency after the fault has occurred in both stationary and non-stationary scenarios.

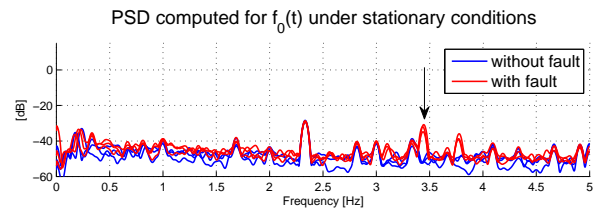

(a) PSD of $f_{0}(t)$ for state A

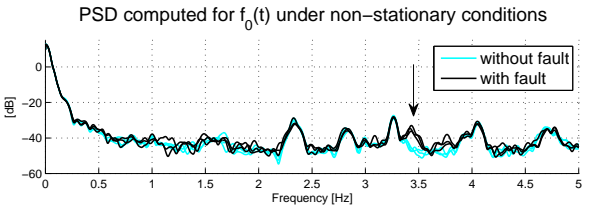

(b) PSD of $f_{0}(t)$ for state $\mathrm{B}$

Fig. 7: PSD of the instantaneous fundamental frequency for stationary and nonstationary operating conditions

\section{Conclusions}

In the first part of the paper, the theoretical tools used to describe electrical three-phase quantities have been presented, as well a proposed set of electrical features that are to be computed. The proposed approach can easily be implemented as a sample-per-sample algorithm, thus making it suitable also for non-stationary signals and on-line monitoring of systems. In the second half of the paper, it has been shown that the proposed method of analysing electrical three-phase signals presents good performance in terms of estimation and tracking of the evolution of the quantities in both stationary and non-stationary conditions. This was achieved by applying the method on electrical three-phase signals measured at the output of the generator, in a complete system simulating a wind turbine. The proposed electrical unbalance indicator has also been shown not to be affected by the non-stationary operating conditions. Moreover, it has been shown that using these estimated features, at least one type of mechanical fault (inner race bearing fault) can be detected.

As future work, this method could be extended to automatically detect more electrical and mechanical faults by further developing the definitions of specific fault indicators. Moreover, by using an adaptive filtering approach for the complex valued filter, the method can be extended for use in an even wider area of applications on electrical three-phase systems operating under non-stationary conditions.

Acknowledgements. This research has been partly supported by KIC InnoEnergy, a company supported by the European Institute of Innovation and Technology (EIT), through KAStrion European project.

The authors would like to thank CETIM (Centre Technique des Industries Mécaniques) for providing the test-bench and experimental data. 


\section{References}

1. Tavner P. J., Offshore wind turbines reliability, availability and maintenance, Institution of Engineering and Technology, London, U.K.(2012)

2. García Márquez F. P., Tobias A. M., Pinar Pérez J. M., Papaelias M.: Condition monitoring of wind turbines: techniques and methods. Renewable Energy, vol. 46, pp 169-178 (2012)

3. Paap G.: Symmetrical components in the time domain and their application to power network calculations. IEEE Transactions on Power Systems, vol. 15, pp. 522528 (2000)

4. Reilly A., Frazer G., Boashash B.: Analytic signal generation-tips and traps. IEEE Transactions on Signal Processing, vol. 42, pp. 3241-3245 (1994)

5. Choqueuse V., Benbouzid M.E.H., Amirat Y., Turri S.: Diagnosis of ThreePhase Electrical Machines Using Multidimensional Demodulation Techniques. IEEE Transactions on Industrial Electronics, vol. 59, pp. 2014-2023 (2012)

6. Granjon P.: Condition monitoring of motor-operated valves in nuclear power plants, in: 8th International Conference on Condition Monitoring and Machinery Failure Prevention Technologies, Cardiff, UK, vol. 1, pp 109-119 (2011)

7. Blodt M., Granjon P., Raison B., Régnier J.: Mechanical fault detection in induction motor drives through stator current monitoring theory and application examples, in: Fault Detection, Wei Zhang (Ed) (2010)

8. Al Ahmar E., Choqueuse V., Benbouzid M.E.H., Amirat Y., El Assad J., Karam R., Farah S.: Advanced signal processing techniques for fault detection and diagnosis in a wind turbine induction generator drive train: A comparative study. Energy Conversion Congress and Exposition (ECCE), IEEE, pp. 3576-3581, Atlanta, GA (2010)

9. Zhang P, Neti P.: Detection of gearbox bearing defects using electrical signature analysis for Doubly-fed wind generators. Energy Conversion Congress and Exposition (ECCE), IEEE, pp. 4438-4444, Denver, CO (2013)

10. Stefani A., Bellini A., Filippetti F.: Diagnosis of Induction Machines' Rotor Faults in Time-Varying Conditions. IEEE Transactions on Industrial Electronics, vol. 56, pp. 4548-4556 (2009)

11. Bouzid M.B.K., Champenois G.: New Expressions of Symmetrical Components of the Induction Motor Under Stator Faults. IEEE Transactions on Industrial Electronics, vol. 60, pp. 4093-4102 (2013)

12. Lyon W. V.: Transient analysis of alternating current machinery - an application of the method of symmetrical components, The Technology Press of MIT and John Wiley \& Sons, New York (1954)

13. Cablea G., Granjon P., Bérenguer C.: Method for computing efficient electrical indicators for offshore wind turbine monitoring. Insight - Non-Destructive Testing and Condition Monitoring, vol. 56, pp. 443-448 (2014)

14. Fortescue C.L.: Method of Symmetrical Co-Ordinates Applied to the Solution of Polyphase Networks. Transactions of the American Institute of Electrical Engineers, vol. XXXVII, pp. 1027-1140 (1918)

15. Boashash B.: Estimating and interpreting the instantaneous frequency of a signal. I. Fundamentals. Proceedings of the IEEE, vol. 80, pp. 520-538 (1992)

16. Welch P.: The use of fast Fourier transform for the estimation of power spectra: A method based on time averaging over short, modified periodograms. IEEE Transactions on Audio and Electroacoustics, vol. 15, pp. 70-73 (1967) 